\title{
Effectiveness of Kinesio Taping for Dynamic Postural Stability in Adolescent Athletes with Chronic Ankle Instability: A Pilot Study
}

\author{
Ji-Hoon Cho' ${ }^{1}$, Seung-Taek Lim² ${ }^{2 *}$, Jupil Ko ${ }^{3 *}$ \\ 1 Shingyeong University, Hwaseong, Republic of Korea \\ ${ }^{2}$ Kangwon National University, Chuncheon, Republic of Korea \\ ${ }^{3}$ Incheon National University, Incheon, Republic of Korea
}

Received: February 8, 2021

Accepted: February 19, 2021

Published online: April 30, 2021

Keywords:

Ankle Stability

Dynamic Balance

Efficacy

Postural Control

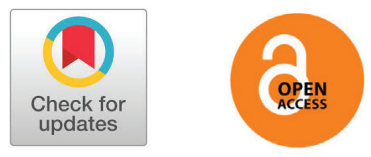

\section{ABSTRACT}

OBJECTIVES To determine the effectiveness of K-tape on dynamic postural stability of the injured limb in adolescent athletes with chronic ankle instability (CAI) by measuring reach distance with the modified Star Excursion Balance Test (mSEBT).

METHODS Twenty volunteers (20 females [17.78 $\pm 1.22 \mathrm{yrs}]$, height [163.89 $\pm 7.69 \mathrm{~cm}]$, mass [62.51 \pm 9.81 $\mathrm{kg}]$ ) with CAI. Four strips of K-tape were applied to the injured limb. Participants completed 4 trials of testing ( 2 with tape, 2 without tape) using the $\mathrm{mSEBT}$ as the assessment tool. We applied 4 strips of K-tape to the injured limb and then tested postural stability using the mSEBT to see if there was an increase of reach distance in the mSEBT. Measuring reach distance in Anterior (AN), Posteromedial (PM), Posterolateral $(\mathrm{PL})$ on the injured limb with K-tape and without K-tape condition. We measured the reach distances and then normalized the distance to account for height differences. A repeated measures t-test was used for this study.

RESULTS There is no statistically significant different between the condition with K-tape and without K-tape in the reach distances on the MSEBT in adolescent athletes with CAI.

CONCLUSIONS Based on the results, there appears to be no improvement of reach distance using the mSEBT in adolescent athletes with CAI. Therefore, clinicians need their thoughtful consideration for applying K-tape to improve dynamic postural stability in adolescent athletes with CAI.

(c) The Asian Society of Kinesiology and the Korean Academy of Kinesiology

\section{Introduction}

A significant amount of research exists describing how ankle instability affects surrounding structures of the ankle [1-7]. Neuromuscular control of the ligaments in the ankle can be lost due to recurrent ankle injuries [2-4]. This then

\section{Correspondence:}

* Jupil Ko, 119 Academy-ro, Yeonsu-gu, Incheon, Republic of Korea 22012; Telephone: 82-32-835-8587; Fax: 82-32-835-0789; Email: jupilko@gmail.com

** Seung-Taek Lim, 1 Kangwondahak-gil, Cheuncheon-si, Gangwon-do, Republic of Korea 24341; Telephone: 82-10-3741-3205; Fax: 82-33-259-5680; Email: limdotor@ gmail.com leads to chronic ankle instability (CAI), which affects postural control [2]. CAI is a term used to categorize a patient with two conditions, mechanical and functional instability of the ankle joint [5]. Mechanical Instability (MI) can be defined as the joint gains more laxity because of structural damage that ligamentous tissues obtain in every ankle sprain $[4,6]$. Functional Instability (FI) is when incidences of repeated joint instability and the feeling of joint instability because the deficits of neuromuscular influence them $[4,6]$. Most ankle sprains develop lateral ligamentous instability because of the 
loss of mechanoreceptors in structures such as tibialis anterior, tibialis posterior, peroneals and the arch, which can then affect balance [1]. Repeated ankle sprains can lead to poor postural stability of the talocrural joint in the ankle [7,8]. Previous research has identified a connection between functional ankle instability and impaired ability to maintain postural control during a single leg stance $[9,10]$.

When a patient sustains an ankle injury, balance and postural stability may be interrupted $[11,12]$. One focus of rehabilitation is on reconditioning the muscles surrounding the injured structures to regain original postural stability and balance $[13,14]$. Oftentimes, a clinician uses balance assessments to return patients to original balance and postural stability $[15,16]$. Balance and postural stability are separated into two categories, static and dynamic control. Static stability requires a stable base of support and minimal body movement, whereas dynamic stability requires movement around the base of support [11]. Dynamic postural stability is a better choice than static for assessment because of the similarities between sport participation and testing with movement [11,17]. One type of dynamic postural stability test is the Star Excursion Balance Test (SEBT). This test requires the subject to maintain his or her center of balance on one foot while reaching as far as possible with the other leg in eight directions [18]. The purpose of the test is to measure the reach distance while the patient maintains a stable base of support while reaching with the opposite limb. Recent research has shown that only three directions of the mSEBT (Modified Star Excursion Balance Test) including posteromedial, posterolateral and anterior direction need to be measured because they are representative of all directions $[19,20]$.

To assist with dynamic postural stability and the star excursion balance test, mechanoreceptors need to be activated. One method to activate mechanoreceptors is the application of K-tape. Placing the tape over certain muscles helps to activate mechanoreceptors, which become less sensitive after acute ankle sprains. According to a study by Seo, et al., K-taping has a positive effect on improving proprioception, repositioning subluxated joints, circulation of blood, correcting muscle function and restoring the function of the muscle [21]. Previous studies with K-tape investigated the proprioceptive aspects of CAI[1]; however, there is limited research involving the use of K-tape on adolescent population to determine how this tape affects the mechanoreceptors in the muscles that support the ankle. The purpose of this study was to determine the effectiveness that K-tape has on postural stability in adolescent population with CAI. We hypothesized that there would be an improvement in postural stability in adolescent population with the application of K-tape when compared to no K-tape application.

\section{Method}

\section{Participants}

The participants for this research study were recruited from the high school soccer program at the Phoenix metropolitan area. An email was sent to the coach assistant to gather all the emails for the individuals in the soccer program. Following the reply to this email, an email was sent to all student-athletes asking for interested participants. Individuals who were interested were given the Cumberland Instability Tool (CAIT) [22] to determine eligibility and asked to sign a consent form. Individuals who met the inclusion criteria signed up for a specific time to complete the testing. Twenty volunteers (20 females [17.65 $\pm 1.22 \mathrm{yrs}]$, height [163.89 $\pm 7.69 \mathrm{~cm}]$, mass $[62.51 \pm 9.81 \mathrm{~kg}])$ were involved in this study. Participants were between 16 and 18 years old and considered recreational athletes by participating in physical activities at least 90 minutes a week to meet the inclusion criteria. Participants who reported $\leq 25$ on the CAIT [22,23], a history of lateral ankle $\operatorname{sprain}(\mathrm{s})$, and a history of "giving way" were only involved in this study [5]. The exclusion criteria included having a recent injury to the lower extremity, surgery in the lower extremity, symptoms of a current lower extremity injury, pregnancy, the diagnosis of a vestibular disorder, Charchot-Marie-Tooth disorder, or other nerve or connective tissue condition [5].

This study was approved by the university's Institutional Review Board (IRB) and all participants were provided an informed consent form prior to testing.

\section{Instruments}

The mSEBT including three reach directions (anterior, posteromedial, posterolateral) was used to assess postural 
stability through reach distance of the participants [24]. In a study by Gribble, et al., the SEBT was originally used as a rehabilitative tool and has been used for over 20 years [11]. In the Gribble study, the reliability of the mSEBT was mentioned over various ranges. When a participant performs the mSEBT multiple times a day, the reliability of this test increases from a range of 0.35-0.84 to a range of 0.81-0.93 [11].

\section{Procedures}

To begin the mSEBT creation, three strips of athletic tape measuring $213 \mathrm{~cm}$ long were cut. The three strips were placed on the floor with the posteromedial (PM) and posterolateral (PL) directions aligned at $135^{\circ}$ from the anterior (AN) direction [25]. The directions were labeled as anterior, posteromedial, and posterolateral. After the placement of the tape, measurements were marked every centimeter after 10 $\mathrm{cm}$ on each strip of tape. Twenty of paper were labeled with either "tape" or "no tape" and folded in half for the participants to choose from.

When the participants arrived for their specific time, they chose their strip of paper and informed the researchers of their first test. Their leg length in injured limb was measured for each participant to normalize for height differences. To take this measurement, the tape measure was placed from the anterior superior iliac spine (ASIS) to the medial malleolus [5]. Next, measurements of the lower leg were taken for the placement of the K-tape. The first measurement was for the peroneal muscle from the lateral surface of the proximal fibula to the plantar aspect of base of 1st metatarsal and medial cuneiform. The next measurement was the tibialis anterior, which was measured from the upper half of the lateral shaft of tibia to the inferior aspect of medial cuneiform and base of 1st metatarsal. The third measurement was taken from the posterior tibialis origin on the fibula and tibia to the navicular. The fourth measurement was of the transverse arch from medial to lateral along the plantar surface of the foot [2]. The length of each measurement was multiplied by $75 \%$ or 0.75 to ensure the tension for each participant was similar despite differences in height [26].

The application of the K-tape was uniform for all participants. One piece was applied over the posterior tibialis muscle, beginning at the posterior borders of the tibia and fibula and extending around the medial malleolus with a moderate amount of tension $[2,26]$. The second strip was applied over the tibialis anterior muscle. The strip began at the upper two-thirds of the anterior surface of the tibia and extended to the first metatarsal on the dorsal surface of the foot. The third strip was placed along the peroneus longus muscle, beginning at the proximal head of the fibula and extending over the lateral malleolus, ending on the plantar surface at the base of the first metatarsal. The final strip of K-tape was placed over the transverse arch of the foot beginning anterior to the medial malleolus along the plantar surface of the foot to the transverse arch. After the application of K-tape, each participant completed the mSEBT twice $[2,26]$. All K-tape applications showed in the $<$ Figure $1>$.
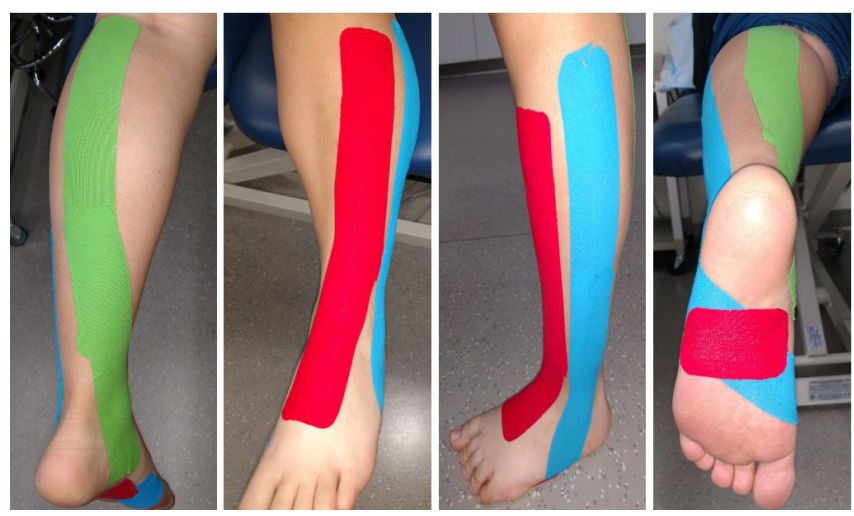

Figure 1. Application of the kinesiology tape

The participants were instructed to stand on their dominant leg with their heel in the middle of the tape star. With their hands on their hips, the participants were instructed to complete the mSEBT starting in the anterior direction. The participants reached with their non-dominant leg as far as they could and lightly tapped on the tape line and returned to the starting position. The distance they reached was recorded before they moved on to the next direction, repeating the reach and continuing around the star; each time coming back to the starting position. Once the first trial was completed, the participants took a 1-minute break and then repeated the test. After the completion of the MSEBT with tape, the participants removed the tape and completed the mSEBT two more times without K-tape. The participants who 
picked the no tape strip first, completed the mSEBT twice then received the tape application and completed the $\mathrm{mSEBT}$ two more times.

\section{Statistical Analysis}

A repeated measures $t$-test was used for this analysis. The statistical software used in this study was Statistical Package for the Social Sciences (SPSS; Version 25.0 for Window, IBM Corporation, Armonk, NY). Data was first entered into an excel spreadsheet, then uploaded into SPSS. The alpha level for this study was $\alpha=.05$.

\section{Results}

No statistically significant difference between with and without K-tape condition in the scores for anterior (AN), posteromedial (PM), posterolateral (PL) reach distance $(p<$ $0.05)$ in the $<$ Table $1>$.

\section{Discussion}

The purpose of this study was to compare the reach distance of adolescent athletes with CAI under a with $\mathrm{K}$-tape and without $\mathrm{K}$-tape condition while performing the mSEBT. The results were calculated using only the anterior, posteromedial, and posterolateral directions based on previous research that indicated that these directions were the most representative of all the directions [19]. The principal finding in the current study was that the application of K-tape was not effective in assisting postural stability in adolescent athletes with CAI, rejecting our hypothesis. Similar to an adult population, there is no statistically significant different between with and without K-tape application in reach distances on the mSEBT [27]. A previous study also shows that K-tape has no effect on muscle activation when compared with white athletic tape and a no tape condition in adult population [28]. However, the results contradict with the research of Nakajima et al which showed that K-tape had a positive effect on dynamic postural control when compared to a placebo group in young adults [26]. In contrast, Nakajima's study showed that K-tape only had a significant effect when applied to female participants. However, they also showed that there was no significant increase in results 10 minutes after the tape application, but there was some increase after 24 hours [26]. There is other previous study that also shows that K-tape has a positive effect on postural stability, however, that research showed that K-tape has been left on for at least 48 hours [2]. However, these studies, which showed the results in contrast to the current study, only included adult population. Therefore, clinicians can recognize that applying K-tape does not increase dynamic postural stability on the mSEBT in adolescent population with CAI.

The current study has some limitation. The first limitation was a small sample size. A larger sample size would allow for fewer varieties to demonstrate statistically significant differences and allow for greater generalization that K-tape is effective in assisting with postural stability. Therefore, the title of the current study include "A Pilot Study". The second limitation was performing both trials with tape and without tape directly after each other, so there was a possibility of a learning effect, which allowed for a better reach with the second attempt. Also, the placebo group need to be included in the future study.

Table 1. Mean ( \pm standard deviation) of $\%$ maximized performance on the mSEBT

\begin{tabular}{llllll}
\hline \multirow{2}{*}{ Reach Direction } & \multicolumn{2}{c}{ mSEBT (\%) } & \multirow{2}{*}{$\boldsymbol{p}$-value } & t-test \\
\cline { 2 - 4 } & without K-tape (mean \pm SD) & K-tape (mean \pm SD) & .667 & -.438 \\
AN & $70.5 \pm 8.7$ & $70.7 \pm 9.1$ & .157 & -1.48 \\
PM & $80.0 \pm 15.3$ & $80.3 \pm 14.9$ & .238 & -1.22 \\
\hline PL & $70.0 \pm 12.1$ & $70.4 \pm 11.6$ & & \\
\hline
\end{tabular}

*Statistically Significantly different between the K-tape and no K-tape $(p<0.05)$ $\mathrm{AN}=$ Anterior; $\mathrm{PM}=$ Posteromedial; $\mathrm{PL}=$ Posterolateral mSEBT $=$ Modified Star Excursion Balance Test 


\section{Conclusions}

Based on the results, there appears to be no statistically significant different between the application of Kinesio tape and an improvement of reach distance using the mSEBT in adolescent athletes. Therefore, clinicians need their thoughtful consideration for applying $\mathrm{K}$-tape to improve dynamic postural stability in adolescent athletes with CAI. The future study should include a larger sample size and the addition of a second application of tape material to determine if $\mathrm{K}$-tape is effective or if any application of tape will improve postural stability.

\section{Acknowledgements}

This work was supported by Incheon National University Research Grant in 2020 (2020-0450).

\section{References}

1. Kim BJ, Lee JH, Kim CT, Lee SM. Effects of ankle balance taping with kinesiology tape for a patient with chronic ankle instability. J Phys Ther Sci. 2015; 27(7):2405-2406.

2. Jackson K, Simon JE, Docherty CL. Extended Use of Kinesiology Tape and Balance in Participants With Chronic Ankle Instability. J Athl Train. 2016; 51(1):1621.

3. Kaminski TW, Hartsell HD. Factors contributing to chronic ankle instability: a strength perspective. J Athl Train. 2002; 37(4):394-405.

4. Freeman MA. Instability of the foot after injuries to the lateral ligament of the ankle. J Bone Joint Surg Br. 1965; 47(4):669-677.

5. Gribble PA, Delahunt E, Bleakley CM, et al. Selection Criteria for Patients With Chronic Ankle Instability in Controlled Research: A Position Statement of the International Ankle Consortium. J Athl Train. 2014; 49(1):121-127.

6. Hertel J. Functional anatomy, pathomechanics, and pathophysiology of lateral ankle instability. J Athl Train. 2002; 37(4):364-375.
7. Isakov E, Mizrahi J. Is balance impaired by recurrent sprained ankle? Br J Sports Med. 1997; 31(1):65-67.

8. Kamali F, Sinaei E, Bahadorian S. The immediate effect of talocrural joint manipulation on functional performance of 15-40 years old athletes with chronic ankle instability: A double-blind randomized clinical trial. J Body Mov Ther. 2017; 21(4):830-834.

9. Konradsen L, Ravn JB. Ankle instability caused by prolonged peroneal reaction time. Acta Orthop Scand. 1990; 61(5):388-390.

10. Ho YH, Lin CF, Chang CH, Wu HW. Effect of ankle kinesio taping on vertical jump with run-up and countermovement jump in athletes with ankle functional instability. J Phys Ther Sci. 2015; 27(7):20872090.

11. Gribble PA, Hertel J, Plisky P. Using the star excursion balance test to assess dynamic postural-control deficits and outcomes in lower extremity injury: a literature and systematic review. J Athl Train. 2012; 47(3):339-357.

12. Zulfikri NMP, Justine MPP. Effects of Kinesio $\left(^{(}\right)$Taping on Dynamic Balance Following Fatigue: a Randomized Controlled Trial. Phys Ther Res. 2017; 20(1):16-22.

13. Lazarou L, Kofotolis N, Pafis G, Kellis E. Effects of two proprioceptive training programs on ankle range of motion, pain, functional and balance performance in individuals with ankle sprain. J Back Musculoskeletal Rehabil. 2018; 31(3):437-446.

14. Mattacola CG, Dwyer MK. Rehabilitation of the Ankle After Acute Sprain or Chronic Instability. J Athl Train. 2002; 37(4):413-429.

15. McKeon PO, Ingersoll CD, Kerrigan DC, Saliba E, Bennett BC, Hertel J. Balance training improves function and postural control in those with chronic ankle instability. Med Sci Sports Exerc. 2008; 40(10):1810-1819.

16. Cote KP, Brunet ME, Gansneder BM, Shultz SJ. Effects of Pronated and Supinated Foot Postures on Static and Dynamic Postural Stability. J Athl Train. 2005; 40(1):4146.

17. de la Motte S, Arnold BL, Ross SE. Trunk-rotation differences at maximal reach of the star excursion balance test in participants with chronic ankle 
instability. J Athl Train. 2014.

18. Mettler A, Chinn L, Saliba SA, McKeon PO, Hertel J. Balance Training and Center-of-Pressure Location in Participants With Chronic Ankle Instability. J Athl Train. 2015; 50(4):343-349.

19. Ko J, Rosen AB, Brown CN. Comparison between single and combined clinical postural stability tests in individuals with and without Chronic Ankle Instability. Clin J Sport Med. 2017; 27(4):394-399.

20. Ko J. Comparison Kinematic Patterns between the Star Excursion Balance Test and Y-Balance Test in Elite Athletes. Korea J Sport Biomech. 2017; 27(3):165-169.

21. Seo HD, Kim MY, Choi JE, et al. Effects of Kinesio taping on joint position sense of the ankle. J Phys Ther Sci. 2016; 28(4):1158-1160.

22. Hiller CE. The Cumberland ankle instability tool: a report of validity and reliability testing. Arch Phys Med Rehabil. 2006; 87(9):1235-1241.

23. Wright CJ, Arnold BL, Ross SE, Linens SW. Recalibration and Validation of the Cumberland Ankle Instability Tool Cutoff Score for Individuals With Chronic Ankle Instability. Arch Phys Med Rehabil. 2014; 95(10):18531859.
24. Hyong IH, Kim JH. Test of Intrarater and Interrater Reliability for the Star Excursion Balance Test. J Phys Ther Sci. 2014; 26(8):1139-1141.

25. Ko J, Wikstrom E, Li Y, Weber M, Brown CN. Performance Differences Between the Modified Star Excursion Balance Test and the Y-Balance Test in Individuals With Chronic Ankle Instability. J Sport Rehabil. 2020; 29(6):748-753.

26. Nakajima MA, Baldridge C. The effect of kinesio tape on vertical jump and dynamic postural control. Int J Sports Phys Ther. 2013; 8(4):393-406.

27. Bailey D, Firth P. Does kinesiology taping of the ankles affect proprioceptive control in professional football (soccer) players?. Phys Ther Sport. 2017; 25:94-98.

28. Briem K, Eythorsdottir H, Magnusdottir RG, Palmarsson R, Runarsdottir T, Sveinsson T. Effects of Kinesio Tape Compared With Nonelastic Sports Tape and the Untaped Ankle During a Sudden Inversion Perturbation in Male Athletes. J Orthop Sports Phys Ther. 2011; 41(5):328-335. 